\section{GP41 SUCCESSFUL UTILIZATION OF DAPTOMYCIN IN TREATING A CHILD WITH ENDOVASCULAR INFECTION. CASE REPORT AND LITERATURE REVIEW}

Hiba El Amin, Alyazia Al Hallami*, Dana Anchassi, Hossam Al Tatari. Tawam hospital, Abu Dhabi/Alain, UAE

\subsection{6/archdischild-2019-epa.107}

Learning objectives Present the successful utilization of daptomycin in a child and provide a literature review on the use of daptomycin in treamtent of MRSA endovascular infection in children

Case summary 13 years old male previously healthy shifted to our hospital from another facility with one week history of right knee trauma after which he developed sepsis and respiratory distress requiring intubation. In our hospital he was found to have bacteremia, septic arthritis, pneumonia and empyema to be due to MRSA. He was also found to have huge emboli in the right pulmonary and right femoral vein which were deemed to be septic emboli. He was initially covered with vancomycin, Gentamicin and Rifampin. The patient later developed Acute Kidney Injury and his Vancomycin levels were eratic and hard to control. His antimicrobial therapy was then shifted to Daptomycin with excellent recovery and outcome.

Methods Case report and literature review

Discussion We believe that this is the first pediatric case in the region to be successfully treated by Daptomycin. In reviewing the literature, use of daptomycin in pediatrics is very limited world-wide. No regional data on its use could be identified through our literature review. The safety, efficacy and pharmacokinetics of daptomycin are not well established in children and therefore using it off-label should be limited to situations when other options are not feasible. In our patient daptomycin was successfully utilized in treatment MRSA endovascular infection without toxicity.

\section{GP42 AUTISM AND VITAMIN A - THREE EYE-CATCHING CASES}

Siobhan Connor*, Catherine Diskin, Louise Kyne, Susan Fitzsimon, Jacqueline McBrien. Temple Street Hospital, Dublin, Ireland

\subsection{6/archdischild-2019-epa.108}

Background We describe 3 cases of young children with Autistic Spectrum Disorder (ASD), who presented with severe visual impairment as a result of vitamin A deficiency, due to highly restrictive diets. A recent study 'Autism Counts' by Sweeney and Staines, found the prevalence rate of ASD of $1.0 \%$ in the Irish population. Vitamin A deficiency is the most common form of malnutrition leading to ocular disease, and the leading cause of childhood blindness worldwide. Vitamin A deficiency is common in the developing world but is a rare occurrence in our population. In developed countries, the diagnosis of malnutrition leading to eye disorders may be missed or delayed due to its rarity.

Ninety percent of children and adolescents with ASD experience more problems with feeding compared to their peers, including a limited food selection often related to aversion and food refusal secondary to sensory disorders.Avoidant/ Restrictive Food Intake Disorder (ARFID) is unfortunately common in children with moderate to severe ASD and therefore these children are vulnerable to developing nutritional deficiencies.

The Cases The 3 cases presented with a combination of symptoms including intolerance of bright lights, blinking, dry eyes, conjunctival redness and parental concerns of reduced vision. They all had severely restricted diets yet 2 of our 3 patients had growth centiles within the normal healthy range. Vitamin A deficiency was diagnosed on ophthalmology examination and supported by biochemical measurement. Examination demonstrated hazy corneas, xerophthalmia, Bitot's spots and irreversible optic atropy, all known signs of vitamin A deficiency. Interestingly skull bony thickening was noted in 2 of the 3 cases. This warrants further explanation as it has yet to be reported in children with vitamin A deficiency.

Treatment included a combination of topical, oral and intramuscular vitamin A administration. Two of our 3 patients have irreversible visual impairment secondary to vitamin A deficiency.

Clinical Lessons From These Cases

Evaluation of a child with ASD should include a detailed nutritional history. Children with a normal weight (or overweight) can have a severe nutrient deficiency. Therefore it is important when taking a nutritional history to include the major food groups, vitamins and nutrients in children with ASD.

Any eye symptom must prompt an urgent ophthalmology assessment.

Consider a visual cause with a change in behaviour or school performance in a child with ASD.

\section{GP43 A CASE OF VAN DER WOUDE SYNDROME}

Spandana Pasupuleti*, Kerry Noy, Jayabharathi Sakamudi. Cwm Taf University Health Board, Merthyr Tydfil, UK

\subsection{6/archdischild-2019-epa.109}

Vander Woude syndrome (VWS) is an autosomal dominant congenital syndrome characterised by a cleft lip or palate and distinctive pits of lower lips. The degree to which individuals carrying the gene are affected widely varies within families.

Introduction VWS comprises of cleft lip or palate and pits in lower lips. The responsible mutation has been identified in interferon regulatory factor 6 gene and the majority of cases have been linked to deletion in chromosome $1 \mathrm{q} 32-\mathrm{q} 41$.

Case report A 3 year old girl born at 36 weeks gestation who initially required admission to special care baby unit for four days for respiratory distress and feeding issues. She was referred to paediatric outpatient at the age of 8 months with chronic cough and noisy breathing. On examination she had noisy breathing, skin dimples on her chest akin to accessory nipples and extra tissue on lower lip resembling pits. She underwent adenotonsillectomy for obstructive sleep apnoea at the age of 2 years. A genetic blood test for Vander Woude syndrome was positive with heterozygous deletion exons 3-9. Her motor delay was improved with physiotherapy but still exhibits speech delay. Intra-oral examination showed eruption of 12 teeth with unusual order and delay in tooth eruption. She is also showing significant behaviour problems as well. The 
child is under multidisciplinary team involving Paediatrics, ENT, Maxillofacial, Genetics and Speech and Language Therapy team.

Discussion VWS is dominantly inherited with prevalence of 1 in 100,000-200,000. However 30 to 50\% of cases arise as denovo mutations hence the diagnosis is made on a clinical basis. Congenital lip pits, cleft lip or palate with varying severity are common presentations in $70 \%$ of cases. Submucous cleft palate is a common feature, nevertheless is easily missed on physical examination. Hypernasal voice and bifid uvula are isolated in VWS. Hypodontia is a cardinal feature of VWS. Extra-oral manifestations are limb anomalies, popliteal webs and brain abnormalities. Accessory nipples, heart defects, and Hirschsprung's have also been reported.

Conclusion Overall affected parents carry a 50\% risk for each child, and more affected phenotypes gives rise to extreme effects in offspring. However, given more mild features are common, it is prudent to always be vigilant for cleft or lip abnormalities in all offspring. These children need multidisciplinary team approach and long-term follow up.

\section{GP44 ERYTHROPOIETIN AS A TREATMENT MODALITY IN HYPERHAEMOLYSIS COMPLICATING SICKLE CELL ANAEMIA}

Ana Louise Hawke*, Helena Conroy, Rosena Geoghegan, Corrina McMahon. Our Lady's Children's Hospital, Crumlin, Dublin, Ireland

\subsection{6/archdischild-2019-epa.110}

Introduction Hyperhaemolysis Syndrome (HS), a severe haemolytic transfusion reaction, is a rare complication in children with Sickle Cells anaemia (SCA) who require transfusion. Donor and autologous red cells are obliterated leading to a worsening of anaemia after transfusion. Erythropoietin has been reported as a treatment modality. This case series examines the experience in our tertiary Paediatric Haematology centre, of treating this haemolytic anaemia with Erythropoietin.

Design and Methods Patients were identified from the SCA patient database at Our Lady's Children's Hospital, Crumlin, Dublin in 2018.

Patient charts were reviewed Details of transfusions, presenting symptoms, examination findings, lab results and treatment modalities were recorded.

Results Three children being treated with erythropoietin following HS were identified.

Patient 1:

Diagnosed with SCA at birth. Transfusion programme started aged 3 years 6 months due to silent infarct on MRI brain.

Transfusion programme stopped aged 3 years 10 months as hyperhaemolysis suspected. Haemoglobin (Hob) fell post transfusion and $\mathrm{HbA}$ rose to $20 \%$ not $60-70 \%$ as would be expected. Autoantibodies were detected.

This was soon complicated by Acute Splenic sequestration with associated high fever. Hob at it's nadir 5.7 g/L. Erythropoetin commenced with good effect to date.

Patient 2:

SCA diagnosed aged 2 years. First transfusion aged 15 years due to crisis with Hob 6. 5 g/L. 5 weeks later admitted with abdominal pain and splenomegaly. Hob $7.45 \mathrm{~g} / \mathrm{L}$ and then fell to $5.7 \mathrm{~g} / \mathrm{L}$. Treated with Immunoglobulin(IVIG) as diagnosis of HS was suspected. Hob A-13.1\%. Commenced on erythropoietin and IV Iron.

2 alloantibodies detected. Hob maintained $>6 \mathrm{~g} / \mathrm{L}$ since then with Erythropoetin 3 times a week and oral iron replacement.

Patient 3:

SCA diagnosed aged 1 year. At age 4 years, 3 admissions with chest crises and associated anaemia.

Hob fell to a nadir of $4.9 \mathrm{~g} / \mathrm{L}$ post transfusion and episodes were treated with IVIG and IV methylprednisolone. Another chest crisis at 4 years 7 months precipitated trial of erythropoietin for 3 months with good effect. No further transfusions required. Erythropoetin restarted aged 6 years 6 months due to $\mathrm{Hob}$ of $5.7 \mathrm{~g} / \mathrm{L}$ in association with infection. Hob improved and patient's Hob now maintained $>6 \mathrm{~g} / \mathrm{L}$ on erythropoietin three times a week.

Conclusion HS is a rare complication of SCA which can cause significant worsening of anaemia that is difficult to treat. Erythropoetin can be used to maintain acceptable levels of haemoglobin to avoid transfusion.

\section{GP45 AUTOIMMUNE ENCEPHALITIS TRIGGERED BY HERPES SIMPLEX VIRUS 1 INFECTION}

${ }^{1}$ Ancuta Bilasco, ${ }^{2}$ Szidonia Florea*, ${ }^{3}$ Ramona Cirt, ${ }^{1}$ Anca Draganescu, ${ }^{1}$ Magda Vasile, ${ }^{1}$ Camelia Kouris, ${ }^{1}$ Cristina Negulescu, ${ }^{4,1}$ Monica Luminos. ${ }^{1}$ National Institute for Infectious Diseases 'Prof. Dr. Matei-Balş, Bucharest, Romania; ${ }^{2}$ Children's Emergency Hospital, ClujNapoca, Romania; ${ }^{3}$ Bucharest Oncology Institute 'Professor Doctor Alexandru Trestioreanu', Bucharest, Romania; ${ }^{4}$ 'Carol Davila' University of Medicine and Pharmacy, Bucharest, Romania

\subsection{6/archdischild-2019-epa.111}

Introduction Herpetic encephalitis is the most frequent central nervous system infection in children caused by viral etiology. It may be a trigger for anti-NMDA receptor (NMDAR) encephalitis with the onset of symptoms usually a few weeks after initial presentation with viral encephalitis.

Case report Approximately one third of the patients with herpetic encephalitis develop anti-NMDAR encephalitis. There are more hypotheses on how anti-NMDAR encephalitis may develop. First, herpes simplex virus 1 (HSV-1) infection causes an inflammatory destruction of neural tissue with subsequent release of neuronal antigens and formation of NMDAR antibodies; or, second, by molecular mimicry. Onset of symptoms is usually after 2-6 weeks after initial infection with HSV 1 . In children symptoms are usually represented by choreoathetosis and orofacial dyskinesias. The diagnosis is made by detecting NMDAR antibodies in cerebrospinal fluid (CSF).

We present the case of an 11 months male infant with no previous medical history, who presented with fever, anorexia, seizures with a focal onset and secondarily generalized, somnolence and progressive neurologic deterioration with onset of symptoms 6 days prior to admission. CSF studies showed a hypertensive, hemorrhagic fluid with low levels of glucose. HSV-1 was detected by real time PCR in CSF. Brain MRI revealed multiple cerebral lesions of different sizes predominantly affecting the right hemisphere, thalamus and basal ganglia with diffuse edema. EEG suggested diffuse cerebral dysfunction. Treatment with Acyclovir was started and continued for 28 days with notable decrease in 